\title{
Ensaio para Medição de Nível e Alcance de Respingos em Soldagem MIG/MAG
}

\author{
(Test for Measurement of Spattering Level and Reach in MIG/MAG Welding)
}

\begin{abstract}
Diandro Bailoni Fernandes ${ }^{1}$, Luiz Carlos de Menezes Júniorl', Louriel Oliveira Vilarinho', Américo Scotti ${ }^{l}$
Universidade Federal de Uberlândia, Faculdade de Engenharia Mecânica, Uberlândia, Minas Gerais, Brasil. diandro@mecanica. ufu.br,luizmenezesjr@mecanica.ufu.br,vilarinho@mecanica.ufu.br,ascotti@ufu.br.
\end{abstract}

Uberlândia-MG

\section{Resumo}

Respingos significam perda de material que deveria ser depositado na junta, além de requerer esforço extra para remoção da superfície da peça. Assim, são indesejáveis e devem ser minimizados. Diferentes abordagens têm sido propostas na literatura para reduzir respingos no processo MIG/MAG. Entretanto, uma primeira pergunta fundamental deve ser respondida: como se quantificar de forma detalhada o nível de respingos em soldagem? Desta forma, este trabalho trata da proposição de um ensaio que consiste basicamente em coletar os respingos gerados durante a realização de cordões de solda feitos com o processo MIG/MAG no modo de transferência metálica por curto-circuito, sob uma condição de soldagem padronizada. A qualificação da geração de respingos é feita em um coletor padrão de respingos, através da separação e coleta do material em três regiões distintas de alcance. De forma quantitativa, este ensaio fornece os índices Rendimento de Deposição, Distribuição Percentual Total por Alcance e Distribuição Percentual por Tamanho de Respingo para cada faixa de alcance.

Palavras-chave: MIG/MAG; Respingos; Curto-circuito.

Abstract: Spattering represents loss of material that should be deposited and requires extra effort for surface cleaning. Spattering is undesired and should be minimized. Different approaches have been proposed in the technical literature for spattering reduction in MIG/MAG process. However, a fundamental question must be answered: how to thoroughly quantify the spattering levels in welding? In this work an experimental test is proposed. It basically consists to collect the spatters generated during the execution of weld beads with the MIG/MAG process in the short-circuit metal transfer mode. The spatter generation qualification is done in a standard spatter collector, through the separation and collection of material in three distinct regions. Quantitatively, this experiment provides the index Deposition Rate, Total Percent Distribution by Reach and Total Percent Distribution by Spatters'Size for each reach range.

Key-words: MIG/MAG; Spattering; Short-circuit.

\section{Introdução}

Respingos significam perda de material que deveria ser depositado na junta, além de requerer esforço extra para remoção da superfície da peça. Assim, são indesejáveis e devem ser minimizados. Diferentes abordagens têm sido propostas na literatura para reduzir respingos no processo MIG/MAG.

Tradicionalmente, a primeira opção para se trabalhar no estudo de respingos na soldagem MIG/MAG se dá pela variação do gás de proteção e observação dos efeitos no rendimento de deposição [1]. A indutância da fonte também é utilizada como fator para se avaliar os resultados na redução dos respingos. Além disto, diferentes abordagens para se estudar os respingos através do estudo da transferência metálica [2] e pelo controle do formato de onda da corrente imposta ao processo [3].

(Recebido em 11/03/2010; Texto final em 17/06/2010). Artigo originalmente publicado no CONSOLDA 2009, Piracicaba, SP, Outubro de 2009.
Apesar de diferentes estudos serem apresentados na literatura [4], uma primeira pergunta fundamental deve ser respondida: como se quantificar de forma detalhada o nível de respingos em soldagem?

Kang et al. [5] propuseram uma caixa que envolve toda a tocha e peça, cuja tampa se move com a tocha. Entretanto, tal abordagem, ao manter o volume constante dentro do sistema, altera a pressão do gás de proteção, além de haver a possibilidade de um respingo ricochetear na parede da caixa e voltar para a poça/arco. Assim, tal sistema possui interferência direta no processo de soldagem. A coleta dos respingos em caixa também é citada no trabalho de Ersoy et al. [6], mas sem fornecer maiores detalhes. Um modelo mais detalhado é apresentado na patente de Kurokawa et al. [7], com a proposição da geometria do corpo de prova de forma a não haver captura de respingos na superfície da peça.

Desta forma, há a necessidade de se propor um ensaio que consiga coletar os respingos gerados durante a realização de cordões de solda e quantificá-los de forma detalhada o alcance, forma e massa. Este ensaio é proposto a seguir numa condição padronizada no modo de transferência metálica por 
curto-circuito, sob uma condição de soldagem padronizada. A escolha da transferência por curto-circuito se dá, pois é aquela utilizada na prática que apresenta maior geração de respingos, se comparada com a transferência spray (goticular).

\section{Materiais e Métodos}

O dispositivo principal da proposição do ensaio é o Coletor de Respingos (Figura 1), cujos principais componentes são descritos como:

- Uma caixa coletora (Figura 2), prismática de cobre, sem a face superior, composta por:

a) Uma placa-base (a) quadrada, de 3,2 $\mathrm{mm}$ de espessura e 534 $\mathrm{mm}$ de lado, formando o fundo da caixa;

b) 4 barras de perfil retangular, sendo 2 maiores (b1), de 508 x 50,8 x 9,5 mm, e 2 menores (b2), de 489 × 50,8 x 9,5 $\mathrm{mm}$, formando as laterais da caixa, aparafusadas entre si e na placa-base através de 5 furos centralizados na lateral em contato com a base, espaçados 101,6 mm entre si;

c) 5 barras de perfil quadrado (c), de 489 x 6,4 x 6,4 mm, atuando como divisórias do espaço interno para fins de determinação de alcance, sendo 4 permanentemente aparafusadas à base e uma central que só é aparafusada de forma provisória à mesma, juntamente com a barra de teste. Estas barras formam regiões para separação de respingos por alcance (até $80 \mathrm{~mm}$, de 81 a $160 \mathrm{~mm}$ e acima de $160 \mathrm{~mm}$ ).

- Um extensor de área de coleta (Figura 3) no formato troncocônico, apelidado de "coifa" (devido à semelhança), também totalmente confeccionado em cobre (folhas de cobre de 0,8 mm de espessura), que é encaixada na base.

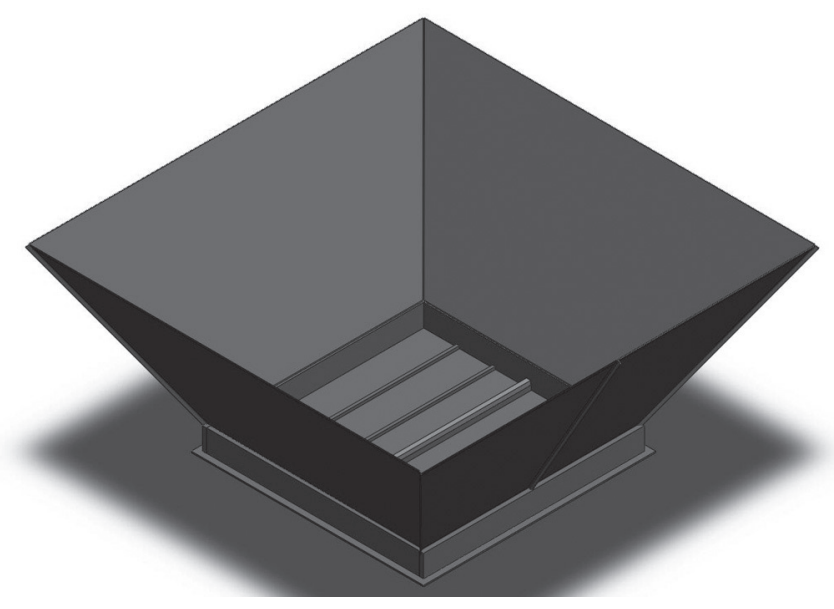

Figura 1. Visão Geral do Coletor de Respingos

Como dispositivo complementar ao experimento, para classificação dos respingos por tamanho, utilizou-se um jogo de três peneiras granulométricas (Figura 4), compostas de telas com espaçamento de 0,50, 1,00 e 2,00 mm, e um prato de fundo (todas de dimensões nominais de $76 \mathrm{~mm}$ de diâmetro por $51 \mathrm{~mm}$ de altura). Para facilitar a operação de separação dos respingos, estas peneiras são fixadas em um agitador mecânico que permite realizar o movimento vibratório de todo o conjunto.

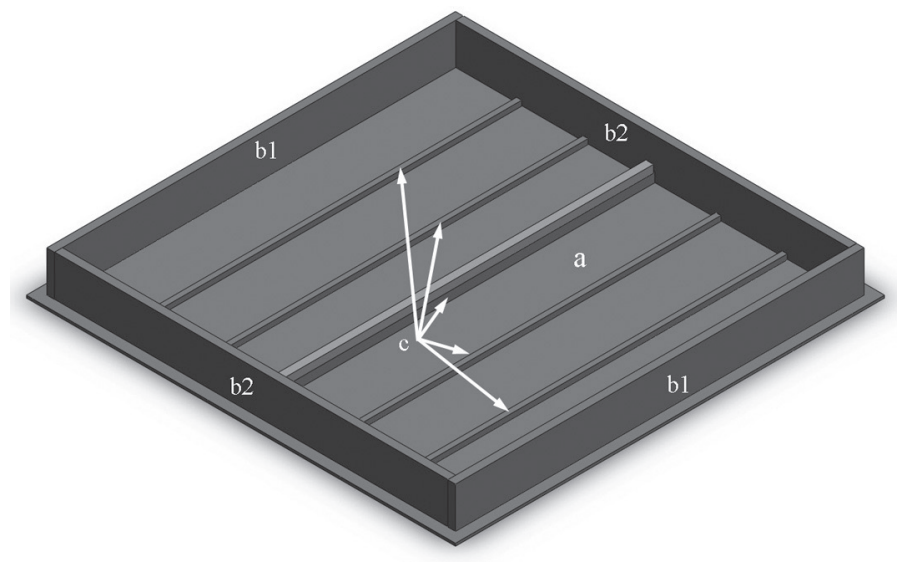

Figura 2. Caixa Coletora (dimensões em mm)

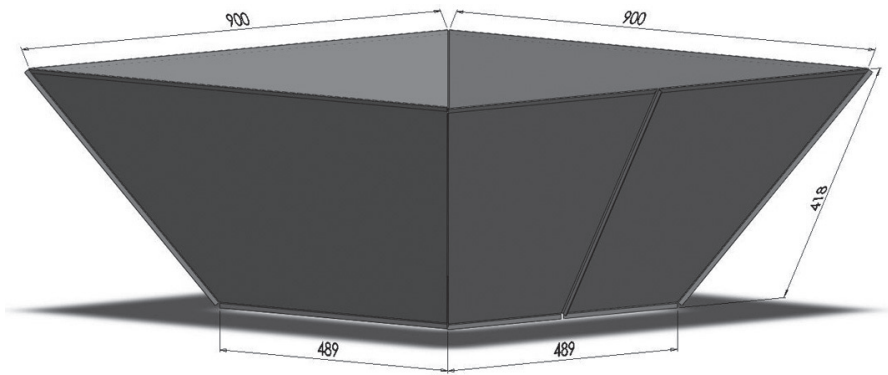

Figura 3. "Coifa" (dimensões em mm)

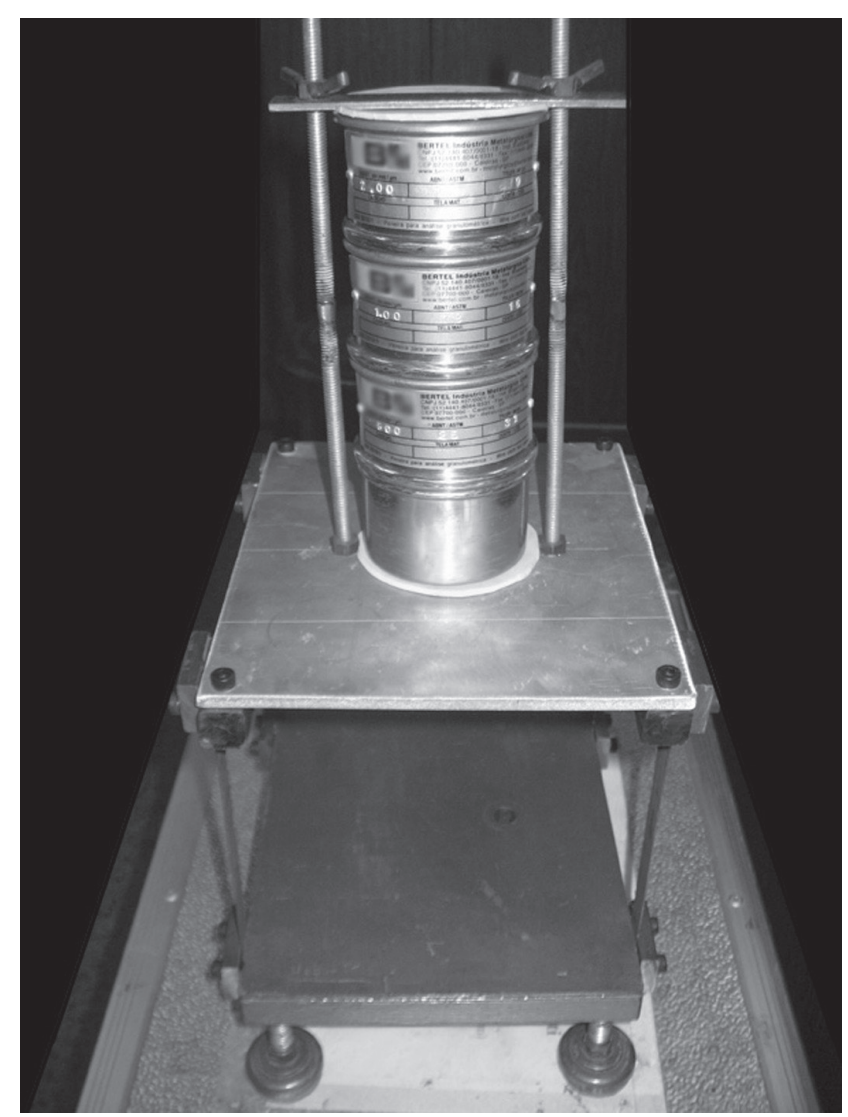

Figura 4. Visão Geral do jogo de peneiras montado em um agitador mecânico 
Para a realização das soldagens-teste, fez-se necessário a utilização de outros equipamentos, como uma fonte de soldagem micro processada do tipo chaveada no secundário, programada para operar no modo MIG/MAG tensão constante, garantindo a mesma regulagem de parâmetros necessários. A tocha de soldagem utilizada foi conectada a uma unidade robótica, visando a repetibilidade da velocidade de soldagem, fixada em $30,0 \mathrm{~cm} / \mathrm{min}$, do comprimento do cordão de solda, de 450 $\mathrm{mm}$, da distância bico de contato-peça (DBCP) em $12 \mathrm{~mm}$ e do correto posicionamento do arame de adição no centro da direção transversal à direção de movimentação da tocha (ao longo do comprimento da barra). O gás de proteção utilizado é o dióxido de carbono, com $99,9 \%$ de pureza, a uma vazão de $151 / \mathrm{min}$. Os arames de adição utilizados são do tipo ER70S-6 cobreados, de duas bobinas diferentes, mas de mesma bitola $(1,20 \mathrm{~mm})$ e de mesmo fabricante.

Um cordão de solda foi depositado por placa de teste, sobre sua superfície oposta à de fixação, em pelo menos 3 barras de teste por bobina testada, descartando pelo menos 5 metros de arame entre cada soldagem, feitas exatamente no centro da barra, em relação à largura, iniciando-se a $15 \mathrm{~mm}$ de uma extremidade e finalizando a $15 \mathrm{~mm}$ da outra (totalizando 450 $\mathrm{mm}$ de comprimento). Para diferentes bitolas de arame, deve-se garantir que todas as poças fundidas tenham aproximadamente o mesmo volume, para permitir comparação entre arames de diferentes bitolas. A partir de uma velocidade de soldagem que proporcionou o volume da poça otimizado para o cordão de $1,2 \mathrm{~mm}$, de forma a preencher toda a largura da barra de teste, definiram-se as correlações entre velocidades de alimentação (Va) e diâmetro de arame (d), como a Equação 1 permite calcular, e tabulado na Tabela 1.

$\frac{V a_{X}}{V a_{1,2}}=\frac{d_{1,2}{ }^{2}}{d_{X}{ }^{2}} \Rightarrow V a_{X}=\frac{7,2}{d_{X}{ }^{2}}$

Tabela 1. Velocidades de Alimentação e de Soldagem padrões para cada bitola de arame

\begin{tabular}{c|c|c}
\hline $\begin{array}{c}\text { Diâmetro do } \\
\text { Arame }[\mathrm{mm}]\end{array}$ & $\begin{array}{c}\text { Velocidade de } \\
\text { Alimentação [m/min] }\end{array}$ & $\begin{array}{c}\text { Velocidade de } \\
\text { Soldagem }[\mathrm{cm} / \mathrm{min}]\end{array}$ \\
\hline 0,6 & 20,0 & 30 \\
\hline 0,8 & 11,3 & 30 \\
\hline 0,9 & 8,9 & 30 \\
\hline 1,0 & 7,2 & 30 \\
\hline 1,2 & 5,0 & 30 \\
\hline
\end{tabular}

As regulagens para as soldagens deverão levar à transferência por curto-circuito. A tensão de referência deve ser regulada para a dada velocidade de alimentação (Tabela 1), de tal forma a se atingir a tensão e corrente médias esperadas, apresentadas na Tabela 2. Para as bobinas utilizadas nos testes, utilizaram-se os valores referentes à bitola de $1,20 \mathrm{~mm}$. Vale ressaltar que apesar da corrente, conseqüentemente calor, ter influência na geração de respingos, seu valor é variado em função da bitola para permitir que com os diversos arames se consigam poças de fusão com o mesmo volume aproximado, considerado neste ensaio como fator mais importante.
Tabela 2. Valores nominais de tensão e corrente padronizados para cada bitola de arame

\begin{tabular}{c|c|c}
\hline $\begin{array}{c}\text { Diâmetro do } \\
\text { Arame } \\
{[\mathrm{mm}]}\end{array}$ & $\begin{array}{c}\text { Faixa de Tensão de } \\
\text { Soldagem Esperada } \\
{[\mathrm{V}]}\end{array}$ & $\begin{array}{c}\text { Faixa de Corrente } \\
\text { Média Esperada } \\
{[\mathrm{A}]}\end{array}$ \\
\hline 0,6 & $\begin{array}{c}\text { (ainda não } \\
\text { especificado) }\end{array}$ & $\begin{array}{c}\text { (ainda não } \\
\text { especificado) }\end{array}$ \\
\hline 0,8 & $\begin{array}{c}\text { (ainda não } \\
\text { especificado) }\end{array}$ & $\begin{array}{c}\text { (ainda não } \\
\text { especificado) }\end{array}$ \\
\hline 0,9 & $\begin{array}{c}\text { (ainda não } \\
\text { especificado) }\end{array}$ & $\begin{array}{c}\text { (ainda não } \\
\text { especificado) }\end{array}$ \\
\hline 1,0 & 19,0 a 21,0 & $165 \pm 5$ \\
\hline 1,2 & 20,0 a 22,0 & $190 \pm 10$ \\
\hline
\end{tabular}

Para evitar adesão de respingos na barra de teste, o que é comum quando utilizado o modo de transferência em questão, o cordão de solda é realizado sobre uma das superfícies de uma barra quadrada de pequena largura, composta de aço ao carbono ABNT 1020 laminadas, de dimensões 480 x 9,5 x 9,5 mm, fixadas à caixa coletora com o uso de parafusos $\mathrm{M} 3 \times \mathrm{x} 0,5 \mathrm{~mm}$, através de furos localizados na superfície oposta à de deposição do cordão, de comprimento e posição detalhados na Figura 5. Cada barra de teste é identificada com o número do teste em questão e tem sua massa obtida antes e após a execução da soldagem, para posterior cálculo do rendimento de deposição, utilizado como parâmetro de controle da estabilidade do processo.

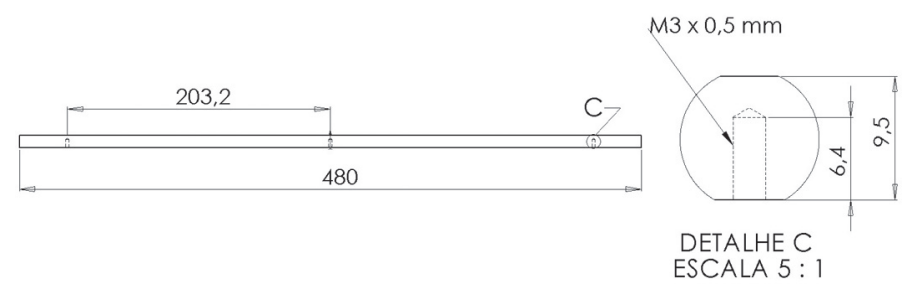

Figura 5. Detalhe das dimensões da barra de teste e de seus furos de fixação.

Nos testes realizados com o uso das bobinas aqui avaliadas, visando demonstrar o funcionamento do ensaio, a tensão foi regulada em 4 níveis diferentes, com variação de 1,0 V entre cada valor, sendo essa faixa e a velocidade de alimentação definida de acordo com a bitola do arame (Tabela 1). A Tabela 3 apresenta os parâmetros de regulagem de tensão para cada teste.

Tabela 3. Identificação dos testes e seus parâmetros

\begin{tabular}{|c|c|c|c|}
\hline Teste & Bobina & $\begin{array}{c}\text { Bitola do } \\
\text { arame }(\mathrm{mm})\end{array}$ & Tensão de Regulagem (V) \\
\hline 1 & \multirow{4}{*}{1} & \multirow{4}{*}{1,20} & 21,0 \\
\hline 2 & & & 22,0 \\
\hline 3 & & & 23,0 \\
\hline 4 & & & 24,0 \\
\hline 5 & \multirow{4}{*}{2} & \multirow{4}{*}{1,20} & 21,0 \\
\hline 6 & & & 22,0 \\
\hline 7 & & & 23,0 \\
\hline 8 & & & 24,0 \\
\hline
\end{tabular}


$\mathrm{Na}$ aquisição dos sinais elétricos - tensão, corrente e velocidade de alimentação - é utilizado um microcomputador com software de aquisição de sinais, uma interface de aquisição A/D USB com resolução de 14 bits, uma interface de proteção e condicionamento dos sinais adquiridos e um tacômetro, conectado ao eixo do motor do empurrador de arame. Para a obtenção das massas inicial e final das barras de teste dos sacos de coleta, para análise quantitativa dos respingos, utilizou-se uma balança de precisão, com capacidade para até $2000 \mathrm{~g}$ e resolução $0,01 \mathrm{~g}$.

Para a coleta dos respingos, foram utilizados sacos plásticos (identificados e com peso de tara determinados) por faixa de alcance (divisórias da caixa coletora) com o auxílio de um ímã, a fim de evitar a coleta de escórias e fuligem. Cada saco foi identificado com o número do teste, alcance e massa inicial do saco, sendo que para alcance sugere-se utilizar letra ou número, facilitando a marcação, como sugerido na Figura 6.

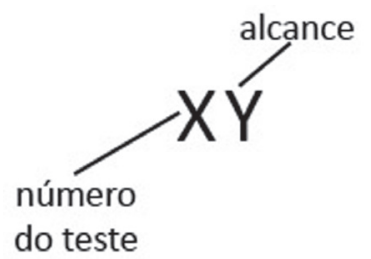

Figura 6. Modelo de identificação do saco para coleta de respingos

$\mathrm{Na}$ determinação da melhor condição experimental dentre os testes realizados, ou seja, qual regulagem de tensão levou à condição de soldagem de melhor regularidade, utilizou-se o Critério Laprosolda para Regularidade de Transferência em MIG/MAG com Curto-Circuito [8], que consiste na análise dos dados obtidos de tensão, corrente e velocidade de alimentação para obtenção de dois parâmetros, a saber:

a) Índice Vilarinho de Regularidade da Transferência em Curtocircuito $\left(\mathrm{IV}_{\mathrm{CC}}\right)$ : é o somatório da relação entre desvio padrão de tempo de arco aberto e tempo de arco aberto e a da relação entre desvio padrão de tempo de curto-circuito e tempo de curto-circuito, dado pela Equação 1. Quanto menor o valor de $\mathrm{IV}_{\mathrm{CC}}$, melhor é a regularidade da transferência metálica.

$I V_{C C}=\frac{\sigma_{t c c}}{t_{c c}}+\frac{\sigma_{t a b}}{t_{a a}}$

b) Faixa admissível de tamanho de gota em transferência por curto-circuito $\left(\Delta \mathrm{F}_{\mathrm{CC}}\right)$ : estabelece a freqüêencia mínima e máxima de curto-circuitos para o tamanho máximo e mínimo da gota admitido, respectivamente, formada na extremidade do arame fundido, em função da velocidade de alimentação $(\mathrm{m} / \mathrm{min})$ e na bitola do arame $(\mathrm{mm})$ utilizado.

Após a realização da análise por este critério, determinouse qual teste para cada bobina apresentou melhor regularidade. Sendo assim, os respingos pertencentes a este teste foram classificados com o uso do separador granulométrico, e novamente armazenados em sacos plásticos com identificação do teste, alcance, região de retenção do separador (peneiras de
0,50, 1,00 e 2,00 mm e prato), e tara. Feito isso, estes sacos de coleta tiveram suas massas obtidas, e através do cálculo a massa de respingos pela diferença entre o valor final e o valor inicial (tara) de cada saco, foi possível determinar as porcentagens coletadas para cada faixa alcance e respectivas porcentagens para cada faixa de tamanho de respingos.

\section{Resultados e Discussão}

A Tabela 4 apresenta os valores médios de tensão, corrente e velocidade de alimentação obtidos para o teste de melhor regularidade, juntamente com os índices pertinentes ao critério e o rendimento de deposição obtido.

Nota-se que os melhores resultados, para cada bobina, foram provenientes de testes com tensões de regulagem diferentes. Mesmo assim, obteve-se valores de $\mathrm{IV}_{\mathrm{CC}}$ praticamente iguais, demonstrando que é possível haver transferência metálica semelhante, no ponto de vista de regularidade, em níveis diferentes de tensão média. Além disso, os valores de rendimento de deposição determinados apresentaram-se muito próximos entre si, demonstrando que a perda de material em forma de respingos foi percentualmente muito semelhante, apesar da frequiência de curtos-circuitos encontrada para o teste 6 ser superior à do teste 3 , justificada pela menor tensão média.

A Tabela 5 apresenta os valores resultantes das massas obtidas com os sacos de coleta com respingos, classificados por alcance e por tamanho, em porcentagem da massa. A coluna Distribuição por Alcance apresenta o total de massa de respingos que atingiu uma determinada distância, enquanto que as colunas abaixo do campo Distribuição por Tamanho trazem, para cada faixa de tamanho de respingo, a porcentagem em massa coletada para o alcance coincidente com a mesma linha na tabela.

A Figura 7 mostra a distribuição percentual de respingos para cada. Nota-se a semelhança entre as quantidades coletadas em cada faixa para ambos os testes, além da redução da quantidade de respingos com o aumento da distância em que este foi coletado em relação à barra de teste.

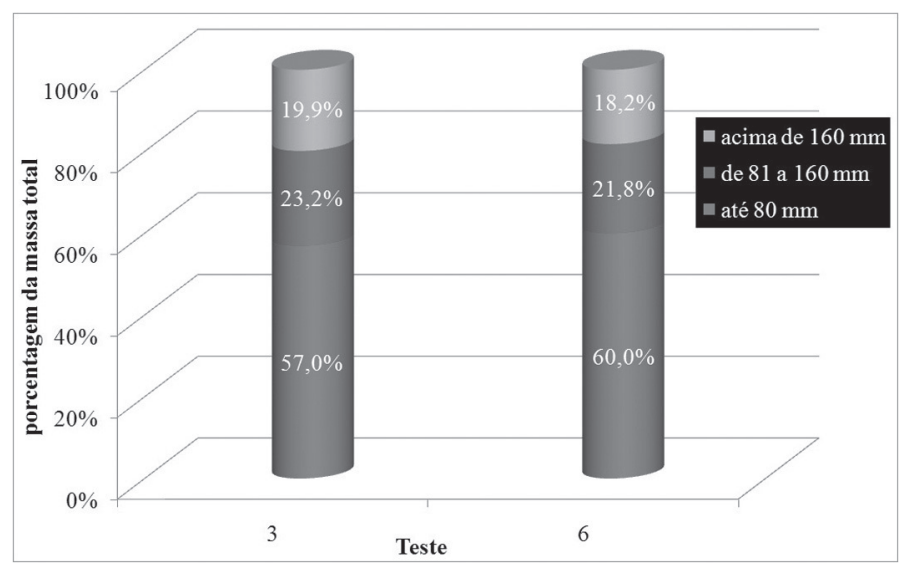

Figura 7. Distribuição dos respingos por alcance, em porcentagem da massa total, nos teste de melhor regularidade de cada bobina 
Tabela 4. Parâmetros Monitorados, índices pertinentes ao Critério de Regularidade

e Rendimento de Deposição da melhor condição paramétrica em cada amostra

\begin{tabular}{c|c|c|c|c|c|c|c|c}
\hline Teste & $\begin{array}{c}\text { Tensão de } \\
\text { Regulagem (V) }\end{array}$ & $\begin{array}{c}\text { Tensão } \\
\text { Média (V) }\end{array}$ & $\begin{array}{c}\text { Corrente } \\
\text { Média (A) }\end{array}$ & $\begin{array}{c}\text { Velocidade de Alimentação } \\
\text { Média (m/min) }\end{array}$ & $\mathrm{IV}_{\mathrm{CC}}$ & $\Delta \mathrm{F}_{\mathrm{CC}}$ & $\mathrm{F}_{\mathrm{CC}}(\mathrm{Hz})$ & $\eta(\%)$ \\
\hline 3 & 23,0 & 20,3 & 191,5 & 5,0 & 0,52 & 53,8 a 78,9 & 28,9 & 98,0 \\
\hline 6 & 22,0 & 19,2 & 190,9 & 5,0 & 0,50 & 53,8 a 78,9 & 36,1 & 97,6 \\
\hline
\end{tabular}

IVCC: Índice Vilarinho de Regularidade da Transferênica em Curto-circuito

$\Delta F_{C C}:$ Faixa de freqüência de curto-circuito para tamanho de gota admissível

$F_{C C}$ : freqüência de curto-circuito encontrada

$\eta$ : rendimento de deposição

Tabela 5. Distribuição dos Respingos para a melhor condição paramétrica de cada amostra

\begin{tabular}{|c|c|c|c|c|c|c|}
\hline \multirow[b]{2}{*}{$\stackrel{\Xi}{\varpi}$} & \multirow[b]{2}{*}{ Alcance } & \multirow[b]{2}{*}{$\begin{array}{l}\text { Distribuição } \\
\text { por Alcance }\end{array}$} & \multicolumn{4}{|c|}{ Distribuição por Tamanho } \\
\hline & & & $\begin{array}{c}\text { Até } \\
0,50 \mathrm{~mm}\end{array}$ & $\begin{array}{c}\text { De } 0,51 \\
\text { a } 1,00 \mathrm{~mm}\end{array}$ & $\begin{array}{c}\text { De } 1,01 \\
\text { a } 2,00 \mathrm{~mm}\end{array}$ & $\begin{array}{l}\text { Acima de } \\
2,00 \mathrm{~mm}\end{array}$ \\
\hline \multirow{3}{*}{3} & até $80 \mathrm{~mm}$ & $57,0 \%$ & $33,7 \%$ & $39,5 \%$ & $26,7 \%$ & $0,0 \%$ \\
\hline & de 81 a $160 \mathrm{~mm}$ & $23,2 \%$ & $57,1 \%$ & $31,4 \%$ & $11,4 \%$ & $0,0 \%$ \\
\hline & acima de $160 \mathrm{~mm}$ & $19,9 \%$ & $80,0 \%$ & $20,0 \%$ & $0,0 \%$ & $0,0 \%$ \\
\hline \multirow{3}{*}{6} & até $80 \mathrm{~mm}$ & $60,0 \%$ & $26,3 \%$ & $56,6 \%$ & $17,2 \%$ & $0,0 \%$ \\
\hline & de 81 a $160 \mathrm{~mm}$ & $21,8 \%$ & $58,3 \%$ & $36,1 \%$ & $5,6 \%$ & $0,0 \%$ \\
\hline & acima de $160 \mathrm{~mm}$ & $18,2 \%$ & $73,3 \%$ & $26,7 \%$ & $0,0 \%$ & $0,0 \%$ \\
\hline
\end{tabular}

A Figura 8 detalha, para cada faixa de alcance, a quantidade percentual de respingos classificada por tamanho para o Teste 3. O comportamento apresentado indica o que já era esperado: que quanto menor o tamanho do respingo, maior será o seu alcance, e seu percentual encontrado é proporcional ao aumento da distância. Para respingos maiores de até $0,50 \mathrm{~mm}$, houve um aumento percentual acompanhando o aumento do alcance, enquanto que para tamanhos superiores à $0,50 \mathrm{~mm}$, a tendência é contrária, ou seja, a diminuição do percentual.

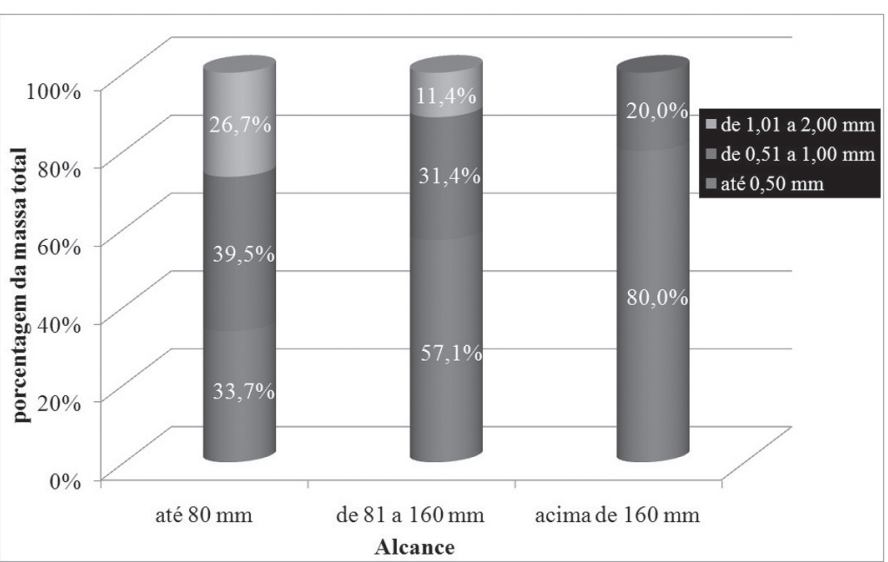

Figura 8. Distribuição dos respingos por tamanho para cada faixa de alcance, referentes ao teste 3
No Teste 6 , de melhor regularidade entre os realizados na bobina 2, o comportamento ocorrido para a distribuição por tamanho de respingos também é muito semelhante ao do teste 3 , como ilustrado pela Figura 9.

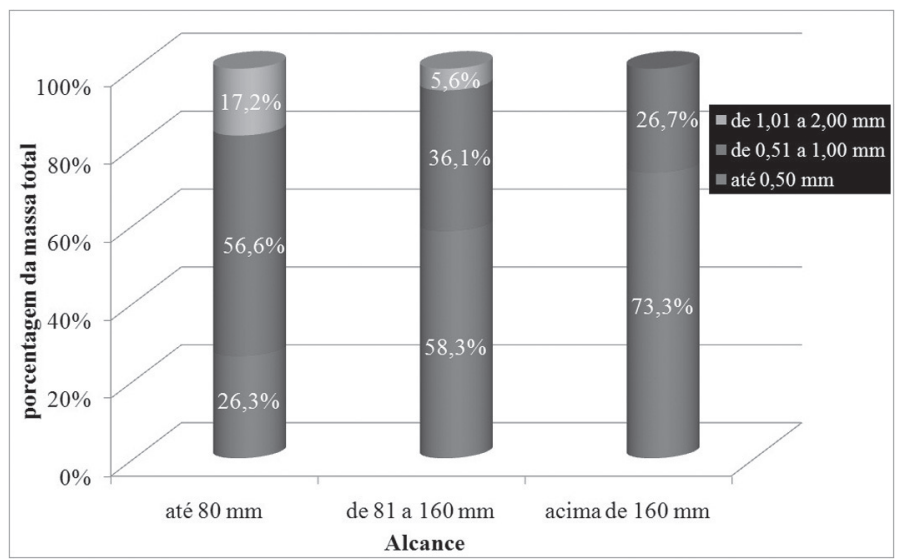

Figura 9. Distribuição dos respingos por tamanho para cada faixa de alcance, referentes ao teste 6

No caso do material testado, não houve ocorrência de respingos de tamanho superior a $2,00 \mathrm{~mm}$, em qualquer faixa de alcance. 


\section{Conclusões}

Com base nos experimentos conduzidos, os quais apresentaram comportamento estatístico regular notado pela boa repetibilidade dos resultados obtidos, conclui-se pela exequibilidade do ensaio proposto. Espera-se que outros pesquisadores possam utilizar o ensaio proposto no estudo e desenvolvimento de processos e condições mais eficientes.

\section{Agradecimentos}

Os autores gostariam de agradecer ao Centro para Pesquisa e Desenvolvimento de Processos de Soldagem - Laprosolda/ UFU, pela infra-estrutura e equipamentos e à Belgo Bekaert Arames pelo fornecimento dos consumíveis. O Prof. Vilarinho gostaria de agradecer também ao CNPq (Processo 473953/20099) e à Fapemig (Processo TEC - APQ-01389-08).

\section{Revisão Bibliográfica}

[1] IKEGAMI, Y. et al. Effects of Shielding Gas for Spatter Reduction of GMAW. Journal of the Japan Welding Society, 75(7), 2006, pp. 570-574.

[2] EICKHOFF, S.T. and EAGAR, T.W. Characterization of Spatter in Low-Current GMAW of Titanium Alloy Plate. Welding Journal, Oct., 1990, 382s-388s.

[3] ERA, T. and UEYAMA, T. Spatter Reduction of GMAW by Current Waveform Control. Journal of the Japan Welding Society, 75(7), 2006, pp. 565-569

[4] YAN, C. et al. Spatter Rate Estimation of GMAW-S based on Partial Least Square Regression. J. Shanghai Jiaotong Univ. (Sci.), 13(6), 2008, pp. 695-701.

[5] KANG, M.J. et al. Spatter Rate Estimation in the ShortCircuit Transfer Region of GMAW. Welding Journal, Sept., 2003, 238s-247s.

[6] ERSOY, U.; HU, S. J. and KANNATEY-ASIBU, E. Observation of Arc Start Instability and Spatter Generation in GMAW. Welding Journal, Feb., 2008, 51s-56s.

[7] KUROKAWA, T. et al. Solid Wire for MAG Welding. United States Patent $\mathrm{N}^{\circ}$ 6,054,675. April 2000, 8p.

[8] SOUZA, D. et al., Relatório Interno Laprosolda/UFU 13/2009 - Manual de Utilização do Programa de Análise de CurtoCircuito para Determinação da Estabilidade de Transferência no Processo de Soldagem MIG/MAG, 9p. 